\title{
Impactos da COVID-19 nas produções das equipes de saúde bucal na atenção
}

\section{primária à saúde}

\author{
Impacts of COVID-19 on the productions of oral health teams in primary health care \\ Impactos del COVID-19 en la producción de equipos de salud bucal en atención primaria de salud
}

Recebido: 20/09/2021 | Revisado: 27/09/2021 | Aceito: 30/09/2021 | Publicado: 02/10/2021

Caroline Dantas Albuquerque Carneiro
ORCID: https://orcid.org/0000-0003-1857-2499
Fundação Oswaldo Cruz, Brasil
E-mail: carol_dantas@ @otmail.com
Sylvia Sampaio Peixoto
ORCID: https://orcid.org/0000-0002-2147-2627
Universidade Federal de Pernambuco, Brasil
E-mail: sampaio_sylvia@ outlook.com

\begin{abstract}
Resumo
Objetivo: analisar as possíveis mudanças e impactos que a pandemia de COVID-19 trouxe para o padrão de produções das Equipes de Saúde Bucal (ESB) da Atenção Primária à Saúde de Pernambuco. Metodologia: trata-se de um estudo descritivo de abordagem quantitativa, com análise comparativa dos registros de procedimentos odontológicos do ano anterior à pandemia de COVID-19 (março de 2019 a março de 2020) e do primeiro ano da pandemia (março de 2020 a março de 2021), através dos dados do Sistema de Informações Ambulatoriais do SUS. Resultados: a quantidade de procedimentos odontológicos no primeiro ano de pandemia foi $75 \%$ menor em relação ao ano anterior. Neste período pandêmico também foi verificada a maior realização de exodontias do que procedimentos restauradores, que revela o quanto ainda prevalece a cultura histórica da população em procurar atendimento apenas nos estágios mais avançados das doenças bucais, somado ao fato de, em diversos períodos do referido ano, os atendimentos odontológicos se restringiram às urgências e emergências. Conclusões: Foi observada uma subutilização da mão de obra e expertise dos profissionais das ESB, que poderiam ter sido melhores aproveitados em serviços "extra-consultórios" durante a pandemia. Espera-se a valorização dos profissionais da odontologia e do programa Brasil Sorridente como um todo, para que a odontologia gratuita e de qualidade chegue a cada vez mais brasileiros.
\end{abstract}

Palavras-chave: COVID-19; Coronavírus; Infecções por Coronavírus; Odontologia; Odontologia em saúde pública.

\begin{abstract}
Objective: to analyze the possible changes and impacts that the COVID-19 pandemic brought to the standard of production of the Oral Health Teams (ESB) of the Primary Health Care of Pernambuco. Methodology: this is a descriptive study with a quantitative approach, with comparative analysis of records of dental procedures from the year prior to the COVID-19 pandemic (March 2019 to March 2020) and the first year of the pandemic (March 2020 to March 2021), using data from the SUS Outpatient Information System. Results: the number of dental procedures in the first year of the pandemic was $75 \%$ lower compared to the previous year. In this pandemic period, tooth extractions were more frequent than restorative procedures, which reveals how much the historical culture of the population still prevails in seeking care only in the more advanced stages of oral diseases, in addition to the fact that, in different periods of the year, dental care was restricted to urgencies and emergencies. Conclusions: An underutilization of the workforce and expertise of the ESB professionals was observed, which could have been better utilized in "out-of-office" services during the pandemic. The appreciation of dental professionals and the Brasil Sorridente program as a all, so that free and quality dentistry reaches more and more Brazilians.
\end{abstract}

Keywords: COVID-19; Coronavirus; Coronavirus infections; Dentistry; Public health dentistry.

\section{Resumen}

Objetivo: analizar los posibles cambios e impactos que la pandemia COVID-19 trajo al estándar de producción de los Equipos de Salud Bucal (ESB) de la Atención Primaria de Salud de Pernambuco. Metodología: se trata de un estudio descriptivo con enfoque cuantitativo, con análisis comparativo de registros de procedimientos dentales del año anterior a la pandemia COVID-19 (marzo de 2019 a marzo de 2020) y el primer año de la pandemia (marzo de 2020 a marzo de 2021). utilizando datos del Sistema de Información Ambulatoria del SUS. Resultados: el número de procedimientos dentales en el primer año de la pandemia fue un $75 \%$ menor en comparación con el año anterior. En este período pandémico, las extracciones dentales fueron más frecuentes que los procedimientos restaurativos, lo que revela cuánto prevalece la cultura histórica de la población en buscar atención solo en las etapas más avanzadas de las enfermedades bucales, además de que, en diferentes períodos de Durante el año, la atención odontológica se limitó a 
urgencias y emergencias. Conclusiones: Se observó una subutilización de la fuerza laboral y la experiencia de los profesionales de la ESB, que podría haber sido mejor aprovechada en los servicios "fuera del consultorio" durante la pandemia. La apreciación de los profesionales de la odontología y del programa Brasil Sorridente en su conjunto, que la odontología gratuita y de calidad llega cada vez a más brasileños.

Palabras clave: COVID-19; Coronavirus; Infecciones por Coronavirus; Odontología; Odontología em salud pública.

\section{Introdução}

Desde sua criação em 1994, ainda com a terminologia de "programa", a Estratégia de Saúde da Família (ESF) visa pôr em prática os preceitos da Atenção Primária à Saúde (APS), com foco no atendimento integral e longitudinal dos pacientes e comunidades, sendo a estratégia orientadora e a principal porta de entrada do Sistema Único de Saúde (SUS) (Dalla Libera, da Costa, Freire \& Brisotti, 2020).

Com foco no princípio da integralidade, no ano de 2004 foi lançado o Programa Brasil Sorridente, via Política Nacional de Saúde Bucal, que veio a dar o acesso a milhões de brasileiros a serviços odontológicos de forma gratuita no SUS. Esses serviços são ofertados em Unidades de Saúde Família (USF) na APS, nas Unidades Odontológicas Móveis (UOM), Centros de Especialidades Odontológicas (CEO) e hospitais (Brasil, 2021).

Com a expansão do Programa, atualmente cerca de 28 mil ESB fazem parte da ESF (Governo do Brasil, 2021) e aproximadamente metade da força de trabalho ocupada em odontologia possui vínculo com o SUS (Gabriel et al., 2020). Na série histórica disponível no site da Secretaria de APS do Ministério da Saúde (MS), a contagem mais recente de cobertura de ESB e de Equipes de Saúde da Família (eSF) é de junho de 2019. No referido período, o estado de Pernambuco possuía 2.242 eSF implantadas, distribuídas por seus 185 municípios, atingindo uma média de cobertura de 90,2\%. As ESB modalidade 1 totalizavam em 1.677 e modalidade 2 em 53, tendo uma média de cobertura de 95,1\% da população do estado. Por ser a porta de entrada do sistema, a ESF tem papel fundamental em casos de surtos e pandemias, como a do novo coronavírus. As eSF oferecem atendimento resolutivo, identificação precoce de casos graves e, quando necessário, o encaminhamento para serviços especializados. Tendo em vista tal envolvimento dos profissionais com os pacientes, a rotina de trabalho nas USF durante tais períodos traz um aumento reconhecido no risco de desenvolver a infecção em questão por parte dos profissionais, principalmente no caso de uma doença de tão fácil e rápida propagação, como a COVID-19 (Japiassu \& Abi Rached, 2020).

A situação das ESB torna-se ainda mais grave, visto que o dentista e sua equipe estão entre os profissionais mais expostos a aerossóis, sendo a principal rota de transmissão do SARSCOV2, agente causador da COVID-19. Considerando a preservação da saúde dos pacientes e da equipe de saúde bucal, em 2020 a Agência Nacional de Vigilância Sanitária (ANVISA) e outras entidades restringiram por diversas vezes os procedimentos odontológicos ao atendimento de urgências e emergências odontológica no SUS, tanto na APS como nos CEO (Carleto \& Santos, 2020).

Tendo em vista tamanha afetação na rotina dos atendimentos odontológicos e as repercussões que o adiamento ou não tratamento de problemas bucais trazem para a saúde e qualidade de vida, principalmente paras as famílias de maior vulnerabilidade socioeconômica que são dependentes do SUS, o presente estudo tem por objetivo analisar as possíveis mudanças e impactos que a pandemia de COVID-19 trouxe para o padrão de produções de procedimentos odontológicos das ESB da APS.

\section{Procedimentos Metodológicos}

Trata-se de um estudo descritivo de abordagem quantitativa, no qual foram analisados comparativamente os registros de procedimentos odontológicos de dois períodos diferentes: 1) ano anterior a pandemia de COVID-19 (março de 2019 a março de 2020), tomando-o como padrão; 2) primeiro ano da pandemia (março 2020 a março 2021). 
Foram utilizados dados secundários provenientes do Sistema de Informações Ambulatoriais do SUS (SIA-SUS) e do relatório de histórico de cobertura da Atenção Básica e de saúde bucal, disponível publicamente no site da Secretaria de Atenção primária à Saúde (www.aps.saude.gov.br).

Os dados do SIA-SUS foram coletados pelo programa Tab para Windows (Tabwin) e exportados para o programa Microsoft Excel versão 2016 (Microsoft Corp., Estados Unidos da América), de maneira a realizar sua consolidação e agrupamento de acordo com o mês e ano de realização dos procedimentos odontológicos.

As variáveis incluídas, coletadas via Tabwin foram: procedimentos realizados, mês de atendimento, frequência e CBO profissional (sendo eles: Cirurgião-Dentista $(C D)$ de saúde da família, CD clínico geral, CD de saúde coletiva e CD da Estratégia de Saúde da Família).

No presente estudo, os principais procedimentos odontológicos realizados pela APS foram divididos nos seguintes grupos: ações coletivas; ações preventivas e educativas individuais (incluindo ART); visitas/atendimentos domiciliares; biópsias; coletas para exames laboratoriais/ acolhimento/ aferição de pressão arterial; testes rápidos; consultas na APS / primeira consulta odontológica programática; atendimento de urgência/ tratamento de abcessos/ lesão em boca/ reimplante dentário; acesso a polpa/ capeamento/ curativo de demora; restaurações; raspagem/ profilaxia; e exodontias.

\section{Resultados}

No ano pré-pandêmico, houve um total de 1.362 .597 procedimentos odontológicos realizados pelas ESB da APS de Pernambuco, dentre os considerados neste estudo. O grupo de procedimentos que mais se destacou no período foi consulta na APS/primeira consulta, sendo responsável por $31,9 \%$ do total, seguido por raspagem/profilaxia $(16,7 \%)$ e restaurações $(13,9 \%)$. Os grupos de procedimentos realizados com menores frequências foram testes rápidos $(0,02 \%)$, coleta para exames laboratoriais/acolhimento/aferição de pressão arterial $(0,5 \%)$ e visitas/atendimentos domiciliares $(0,7 \%)$.

Nos primeiros 12 meses de pandemia no Brasil, a produção das ESB da Atenção Básica caiu 75,6\% em relação ao ano anterior, totalizando 332.309 procedimentos odontológicos. Os procedimentos que apareceram com maior frequência foram consulta na APS/primeira consulta (19,7\%), raspagem e profilaxia (16,9\%) e exodontias (13,9\%). Os procedimentos que tiveram menor produção foram biópsias $(0,03 \%)$, visitas/atendimentos domiciliares $(0,8 \%)$ e coleta para exames laboratoriais/acolhimento/aferição de pressão arterial (1\%).

Os detalhes que quantidade total e percentual de cada grupo de procedimentos odontológicos realizados no período pré e pandêmico estão na Tabela 1 abaixo. 
Tabela 1 - Procedimentos odontológicos realizados na APS de Pernambuco no ano pré e pandêmico.

\begin{tabular}{|c|c|c|c|c|}
\hline Grupo de procedimentos & $\begin{array}{l}\text { Março/2019 - } \\
\text { março/2020 }\end{array}$ & & $\begin{array}{l}\text { Março/2020 - } \\
\text { março/2021 }\end{array}$ & \\
\hline & $\mathrm{n}$ & $\%$ & $\mathrm{n}$ & $\%$ \\
\hline Ações coletivas & 103.977 & 7,6 & 32.944 & 9,9 \\
\hline $\begin{array}{l}\text { Ações preventivas e educativas } \\
\text { individuais (incluindo ART) }\end{array}$ & 142.350 & 10,4 & 40.397 & 12,1 \\
\hline Visitas/atendimentos domiciliares & 9.039 & 0,7 & 2.515 & 0,8 \\
\hline Biópsias & 59 & 0,004 & 113 & 0,03 \\
\hline $\begin{array}{l}\text { Coleta p/ exames laboratoriais + } \\
\text { acolhimento + aferição PA }\end{array}$ & 6.413 & 0,5 & 3.452 & 1 \\
\hline Testes rápidos & 306 & 0,02 & 98 & 0,03 \\
\hline Consulta APS $+1^{\mathrm{a}}$ consulta & 434.244 & 31,9 & 65.544 & 19,7 \\
\hline $\begin{array}{l}\text { Urgência + abcessos + lesão em } \\
\text { boca + reimplante }\end{array}$ & 30.501 & 2,2 & 15.988 & 4,8 \\
\hline $\begin{array}{l}\text { Acesso a polpa + capeamento + } \\
\text { curativo }\end{array}$ & 105.784 & 7,8 & 40.361 & 12,1 \\
\hline Restauração & 189.656 & 13,9 & 28.521 & 8,6 \\
\hline Raspagem + profilaxia & 228.123 & 16,7 & 56.183 & 16,9 \\
\hline Exodontia & 112.145 & 8,2 & 46.193 & 13,9 \\
\hline TOTAL & 1.362.597 & 100 & 332.309 & 100 \\
\hline
\end{tabular}

Fonte: Sistema de Informações Ambulatoriais do SUS (SIA-SUS).

\section{Discussão}

Apesar da considerável expansão da rede SUS e do quantitativo de ESB na APS, estudo revela que de 2015 a 2017 houve considerável diminuição na implantação de ESB, da cobertura, do financiamento e do quantitativo de primeira consulta odontológica (Chaves et al., 2017). Há esforços para tentar tornar o programa Brasil Sorridente uma estratégia que tenha permanente expansão, porém o que vem acontecendo é o contrário, com o desfinanciamento e desmonte, a exemplo da Emenda Constitucional $n^{\circ} 95$ (EC 95), que reduz por duas décadas os recursos para educação e saúde, e da nova Política Nacional de Atenção Básica de 2017, que deixa as ESB como “complementares” às eSF, além de permitir a formação de ESB sem Auxiliares de Saúde Bucal (ASB) (Narvai, 2017; Carletto \& Santos, 2020).

Ainda nos dias atuais, o atendimento odontológico é marcado por ações curativas e/ou mutiladoras e a formação dos CD ainda é centrada no modelo biologicista, voltada para espaços privados, modelo que acaba sendo também reproduzido nos serviços públicos (Carletto \& Santos, 2020; Martelli et al., 2010). No Brasil é historicamente verificada uma busca tardia aos serviços odontológicos para solução das doenças bucais. A procura de atendimento, motivado por dor em caso de emergência, ocorre principalmente em estágios mais avançados dessas doenças, o que resulta ocasionalmente na extração dentária (SilvaJúnior, Sousa, Batista \& Sousa, 2017). Esta tomada de decisão por parte do paciente deve-se, além do custo, à sensação de alívio sintomatológico e possibilidade de novos quadros de dor (Alomari, Khalaf \& Al-Shawaf, 2013). 
Percebe-se que a extração dentária é um procedimento bastante prevalente nos boletins de produção odontológica, este que deveria ser o último procedimento de escolha para resolução de problemas bucais. Tal rotina de escolha pela extração dentária, na crença de remoção da causa, interferindo diretamente no desconforto e sintomatologia dolorosa, foi um dado verificado no presente estudo, onde observa-se a realização de mais exodontias $(13,9 \%)$ do que restaurações $(8,6 \%)$ no ano pandêmico.

Por tratar diretamente as vias respiratórias superiores dos pacientes, os profissionais da odontologia foram considerados vulneráveis à contaminação pelo novo coronavírus. Mesmo com todos os EPI necessários, os equipamentos de trabalho produzem aerossóis que permanecem no ar por até algumas horas, o que pode inclusive gerar a contaminação cruzada de outros pacientes (Vicente, Silva, Barbosa, Pinheiro \& Leite, 2020).

Por isso, por diversas vezes durante a pandemia, foi orientada e até determinada a suspensão dos atendimentos eletivos e limitá-los a atendimentos de urgências e emergências odontológicas. Em 12 de março de 2020 Pernambuco notificou o primeiro caso de COVID-19 no estado e já a partir do dia 20 do mesmo mês a Secretaria Estadual de Saúde (SES) determinou a suspensão da realização de cirurgias eletivas, consultas e procedimentos diagnósticos ambulatoriais em todas as unidades da rede assistencial pública e privada em todo o Estado, pela portaria n 107/2020 (Pernambuco, 2020a). Já no início de maio foi lançado o decreto estadual 49.017, que intensificava as medidas restritivas, inclusive com controle de circulação, de 16 a 31 de maio (Pernambuco, 2020b). O Conselho Regional de Odontologia (CRO) também lançou recomendações quanto à restrição de atendimentos eletivos (Resolução $n^{\circ}$ 01/2020) e cartilhas que traziam os cuidados e limpezas com o ambiente de atendimento, inclusive com a orientação de intervalo de 30 minutos entre o atendimento de um paciente e outro (Resolução ${ }^{\circ}$ 03/2020).

Apesar de em junho de 2020 a SES autorizar o retorno aos atendimentos eletivos no setor público e privado (Portaria 208/2020), poucos dias depois o MS orienta a suspensão procedimentos eletivos e o remanejamento do trabalho da ESB, realocando-as para os fast-track, realização de testes rápidos e coleta de material biológico por meio de Swab (Nota técnica ${ }^{\circ}$ 16/2020). Em novo manual de orientações necessárias para retorno das atividades eletivas no SUS, lançado em novembro de 2020 pelo CRO, a entidade recomenda que sejam evitados procedimentos com caneta de alta e baixa rotação, ultrassom e jato de bicarbonato, seringa tríplice, uso da cuspideira, além da adoção de tecnologias para que as ações de promoção da saúde e prevenção de agravos sejam de modo não presencial. Com novo pico de contaminação e mortes no estado, novamente em março de 2021 a SES recomenda a suspensão de consultas e procedimentos eletivos no Estado (Portaria 187/2021).

Devido aos diversos períodos de paralisação dos atendimentos, além do controle de circulação da população em geral e a falta de EPI adequados e necessários para a proteção dos trabalhadores/trabalhadoras e usuários/usuárias, observa-se a enorme discrepância no total de procedimentos odontológicos realizados no ano pré-pandêmico para o ano pandêmico, que totalizou mais de um milhão de procedimentos a menos, além da queda de 12,2\% nas consultas na APS e primeiras consultas odontológicas programáticas no ano da pandemia. Também destaca-se que por um período considerável de tempo só estava autorizado a realização de atendimentos de urgência e emergência, o que pode explicar o fato de $30,8 \%$ dos atendimentos do período de pandemia ter sido urgências, abcessos, lesões em boca, reimplante dentário, acessos a polpa, capeamentos, curativos e exodontias.

Diante de tal restrição e cuidados com os procedimentos odontológicos que mais podem colaborar na transmissão da COVID-19, que são aqueles geradores de aerossóis, foi esperada a redução na execução de alguns procedimentos, como, por exemplo, as restaurações, que tiveram uma diminuição percentual de 5,3\%, caindo da terceira para a sétima colocação, redução essa que pode ter corroborado com a postergação tratamento e agravamento do quadro do de problemas bucais, como a cárie que quando não tratada pode evoluir para um abscesso. 
Porém, contrariando o esperado, nos dois períodos analisados, as raspagens e profilaxias se mantiveram ocupando o segundo lugar de procedimentos mais realizados e ainda tiveram um discreto aumento percentual (de 0,2\%) no ano 2020-2021. Por serem procedimentos que geram aerossóis, principalmente quando a raspagem é realizada com equipamentos de ultrassom, esperava-se a redução da execução dos mesmos.

Tomando como referência as notas e portarias do MS, do Conselho Federal de Odontologia, do CRO e outras entidades que recomendavam a realocação das ESB, esperava-se que os procedimentos mais executados no ano de pandemia fossem as ações preventivas e educativas individuais, uma maior participação das ESB em coletas para exames laboratoriais, no acolhimento, aferindo pressão arterial dos pacientes, executando testes rápidos, além de uma maior frequência das visitas e consultas domiciliares. Realmente o aumento, em participação percentual do total de procedimentos, aconteceu com todos, porém de maneira discreta e no ranking geral apenas as ações educativas individuais que aparecem entre os cinco primeiros colocados, estando, nos dois anos, na quarta colocação.

A integração da ESB nas eSF ainda é um desafio. A formação dos profissionais de odontologia pautada no modelo biomédico colabora para que os mesmos optem por trabalhar basicamente nas consultas clínicas, raramente se envolvendo em ações conjuntas com a eSF, com as famílias e a comunidade. Faz-se necessário a quebra de tal isolamento da ESB e que os profissionais busquem a troca de conhecimentos, o reconhecimento de habilidades e competências de cada membro da eSF, se fazendo presente na construção coletiva de intervenções, para que haja uma atuação consoante com os princípios e diretrizes da ESF (Scherer, Scherer, Chaves \& Menezes, 2018).

Tendo em vista a evitação em se fazer aglomerações, com o objetivo de diminuir a propagação do vírus, presumia-se uma queda na produção de ações e orientações coletivas. Mas, ao contrário do esperado, tais ações saíram da sétima para a sexta colocação de procedimentos mais realizados, tendo um aumento de 2,3\% na participação anual no ano pandêmico. $\mathrm{O}$ que pode justificar tal aumento é a inserção de tecnologias na rotina de trabalho, como, por exemplo, encontro de grupos e orientações coletivas via vídeo chamadas online, como orientado pelo manual do CRO lançado em novembro de 2020.

As biópsias, apesar de serem os procedimentos menos realizados nos dois períodos analisados, também tiveram um discreto aumento no ano pandêmico. Destaca-se que a incidência de câncer de cabeça e pescoço vem aumentando significativamente desde a última década e que aproximadamente $40 \%$ dos casos de câncer dessas regiões ocorrem na cavidade oral (Da Silva et al., 2020). Logo, é necessário que os gestores das três esferas de governo dêem atenção e invistam na capacitação dos profissionais da ESB na APS para que trabalhem na prevenção e detecção precoce de tais problemas.

\section{Considerações Finais}

A pandemia de COVID-19 trouxe mudanças no modo de viver das populações e nas práticas de saúde. Os consultórios odontológicos foram diretamente afetados, visto que foram considerados lugares de alto risco de contaminação. Apesar disso, os profissionais da odontologia foram os de menor índice de contaminação dentre os profissionais de saúde, segundo relatório do MS e CFO, mostrando como tal categoria se preocupa e trabalha pautada nas normas de biossegurança.

Apesar de demonstrarem alto nível de cuidado, conhecimentos e práticas de biossegurança, os profissionais das ESB se encontram com um campo de atuação limitado e com pouco aproveitamento na APS neste período de pandemia, além de sofrerem com os sucessivos desfinanciamentos e desmonte da Política Nacional de Saúde Bucal.

Espera-se a valorização dos profissionais da odontologia, da contribuição que os mesmos trazem para a saúde e o bem viver das populações e que o Brasil Sorridente caminhe no sentido de sua evolução, com o aumento da inserção das ESB nas eSF e, consequentemente, do acesso da população brasileira à uma odontologia gratuita e de qualidade. 


\section{Referências}

Alomari, Q. D., Khalaf, M. E., \& Al-Shawaf, N. M. (2013). Relative contribution of restorative treatment to tooth extraction in a teaching institution. Journalof oral rehabilitation, 40(6), 464-471.

Brasil. Ministério da Saúde (2017). Portaria/MS n 2.436. Aprova a Política Nacional de Atenção Básica, estabelecendo a revisão de diretrizes para a organização da Atenção Básica, no âmbito do SUS. Diário Oficial da União 2017; 10 jun.

Brasil. Ministério da Saúde (2020). Nota técnica n 16/2020. Diário Oficial da União, Brasília-DF, 17 de junho de 2020.

Brasil. Ministério da Saúde, Secretaria de Atenção Primária à Saúde (2021). Brasil Sorridente - Política Nacional de Saúde Bucal. <https://aps.saude.gov.br/ape/brasilsorridente>.

Carletto, A. F., \& Santos, F. F. D. (2020). A atuação do dentista de família na pandemia do Covid-19: o cenário do Rio de Janeiro. Physis: Revista de Saúde Coletiva, 30, e300310.

Chaves, S. C. L., Almeida, A. M. F. D. L., Rossi, T. R. A., Santana, S. F. D., Barros, S. G. D., \& Santos, C. M. L. (2017). Política de Saúde Bucal no Brasil 2003-2014: cenário, propostas, ações e resultados. Ciência \& Saúde Coletiva, 22, 1791-1803.

Conselho Regional de Odontologia de Pernambuco (2020a). Resolução n 01/2020. Recife, 18 de maio de 2020.

Conselho Regional de Odontologia de Pernambuco (2020b). Resolução n 03/2020. Recife, 08 de junho de 2020.

Conselho Regional de Odontologia de Pernambuco (2020c). Manual com orientações necessárias para retorno das atividades eletivas no SUS. Recife, 11 de novembro de 2020 .

da Silva, F. A., Roussenq, S. C., de Souza Tavares, M. G., de Souza, C. P. F., Mozzini, C. B., Benetti, M., \& Dias, M. (2020). Perfil epidemiológico dos pacientes com câncer de cabeça e pescoço em um centro oncológico no sul do Brasil. Revista Brasileira de Cancerologia, 66(1).

Dalla Libera, T. C., da Costa, R. F. A., Freire, G. C., \& Brisotti, J. L. (2020). Análise comparativa entre estratégia de saúde da família e unidade básica de saúde na referência ao nível assistencial secundário em cardiologia. Manuscripta Médica, 3, 60-68.

Gabriel, M., Cayetano, M. H., Chagas, M. M., Araujo, M. E. D., Dussault, G., Pucca Junior, G. A., \& Almeida, F. C. S. D. (2020). Mecanismos de ingresso de dentistas no SUS: uma agenda prioritária para o fortalecimento do Brasil Sorridente. Ciência \& Saúde Coletiva, 25, 859-868.

Governo do Brasil. Saúde (2021). Número de equipes de saúde bucal cresceu em 2020. 14 de janeiro de 2021. <https://www.gov.br/pt-br/noticias/saude-evigilancia-sanitaria/2021/01/numero-de-equipes-de-saude-bucal-cresceu-em-2020>.

Japiassu, R. B., \& Abi Rached, C. D. (2020). Como a Estratégia de Saúde da Família pode ser considerada ferramenta de apoio no combate ao COVID-19?

Martelli, P. J. D. L., Macedo, C. L. S. V., Medeiros, K. R. D., Silva, S. F. D., Cabral, A. P. D. S., Pimentel, F. C., \& Monteiro, I. D. S. (2010). Perfil do cirurgião-dentista inserido na Estratégia de Saúde da Família em municípios do estado de Pernambuco, Brasil. Ciência \& Saúde Coletiva, $15,3243-3248$.

Narvai, P. C. (2017). O fim do Brasil Sorridente? Jornal Odonto. Brasil, n. 238, 17 ago. <https://www.jornaldosite.com.br/materias/artigos\&cronic as/anteriores/paulo\%20capel/artcapel238.htm>.

Pernambuco. Secretaria Estadual de Saúde (2020a). Portaria n 107/2020. Recife, 23 de março de 2020.

Pernambuco. Governo do Estado (2020b). Decreto n 49.017. Recife, 11 de maio de 2020.

Pernambuco. Secretaria Estadual de Saúde (2020c). Portaria n ${ }^{\circ}$ 208/2020. Recife, 10 de junho de 2020.

Pernambuco. Secretaria Estadual de Saúde (2021). Portaria n 187/2021. Recife, 17 de março de 2021.

Ribeiro, L. M. C. A. V., Ferreira, M. M., Lima, J. G. C., Santos, A. A., Medeiros, C. K. S., Almeida, D.R. M. F., Gonçalves, G. C., Silva, H. F. V., Araújo, S. L. S., Pinheiro, J. C., Leite R.B., \& Oliveira, R. D. B. (2021). O impacto da pandemia do COVID-19 no atendimento odontológico infantojuvenil no Sistema Único de Saúde de João Pessoa - PB. Research, Society and Development, v. 10, n. 5.

Scherer, C. I., Scherer, M. D. D. A., Chaves, S. C. L., \& Menezes, E. L. C. D. (2018). O trabalho em saúde bucal na Estratégia Saúde da Família: uma difícil integração? Saúde em Debate, 42, 233-246.

Silva-Junior, M. F., Sousa, A. C. C. D., Batista, M. J., \& Sousa, M. D. L. R. D. (2017). Condição de saúde bucal e motivos para extração dentária entre uma população de adultos (20-64 anos). Ciência \& Saúde Coletiva, 22, 2693-2702.

Vicente, K., Silva, B. M. D., Barbosa, D. D. N., Pinheiro, J. C. P., \& Leite, R. B. (2020). Diretrizes de biossegurança para o atendimento odontológico durante a pandemia do COVID-19: revisão de literatura. Revista Odontológica de Araçatuba, 41(3), 29-32. 